\title{
A novel test data compression approach based on bit reversion
}

\author{
Shuo Cai ${ }^{1 a)}$, Yinbo Zhou ${ }^{2}$, Peng Liu ${ }^{2}$, Fei Yu ${ }^{1}$, and Wei Wang ${ }^{1}$ \\ ${ }^{1}$ Hunan Provincial Key Laboratory of Intelligent Processing of Big Data on \\ Transportation \& College of Computer and Communication Engineering, Changsha \\ University of Science and Technology, China \\ ${ }^{2}$ College of Information Science \& Engineering, Hunan University, China \\ a)caishuo@csust.edu.cn
}

\begin{abstract}
Test data compression is an effective methodology for reducing test data volume and testing time. This paper presents a new test data compression approach based on bit reversion, which compresses data more easier by reversing some test data bits without changing the fault coverage. As there are some don't care bits in test set, when they are filled, many faults will be repeatedly detected with multiple vectors. Correspondingly, a lot of bits in the test set can be modified without affecting the fault coverage. Experimental results show that the proposed method can increase compression ratio of code-based schemes by around $10 \%$.
\end{abstract}

Keywords: test data compression, code, bit reversion

Classification: Integrated circuits

\section{References}

[1] U. S. Mehta, et al.: "Run-length-based test data compression techniques: How far from entropy and power bounds? - A survey," VLSI Design 2010 (2010) 670476 (DOI: 10.1155/2010/670476).

[2] P. T. Gonciari, et al.: "Improving compression ratio, area overhead and test application time for system-on-a-chip test data compression/decompression," Design, Automation \& Test in Europe Conf. (2002) 604 (DOI: 10.1109/DATE. 2002.998363).

[3] Z. You, et al.: "A scan disabling-based BAST scheme for test cost reduction," IEICE Electron. Express 8 (2011) 1367 (DOI: 10.1587/elex.8.1367).

[4] A. Jas, et al.: "An efficient test vector compression scheme using selective Huffman coding," IEEE Trans. Comput.-Aided Design Integr. Circuits Syst. 22 (2003) 797 (DOI: 10.1109/TCAD.2003.811452).

[5] X. Kavousianos, et al:: "Optimal selective Huffman coding for test-data compression,” IEEE Trans. Comput. 56 (2007) 1146 (DOI: 10.1109/TC.2007. 1057).

[6] P. Sismanoglou and D. Nikolos: "Input test data compression based on the reuse of parts of dictionary entries: Static and dynamic approaches," IEEE Trans. Comput.-Aided Design Integr. Circuits Syst. 32 (2013) 1762 (DOI: 10. 1109/TCAD.2013.2270433).

[7] T. B. Wu, et al.: "Efficient test compression technique for SOC based on block merging and eight coding," J. Electron. Test. 29 (2013) 849 (DOI: 10.1007/ s10836-013-5415-7). 
[8] A. Chandra and K. Chakrabarty: "System-on-a-chip test-data compression and decompression architectures based on Golomb codes," IEEE Trans. Comput.-Aided Design Integr. Circuits Syst. 20 (2001) 355 (DOI: 10.1109/43. 913754).

[9] A. Chandra and K. Chakrabarty: "Test data compression and test resource partitioning for system-on-a-chip using frequency-directed run-length (FDR) codes,” IEEE Trans. Comput. 52 (2003) 1076 (DOI: 10.1109/TC.2003. 1223641).

[10] P. T. Gonciari, et al.: "Variable-length input Huffman coding for system-on-achip test," IEEE Trans. Comput.-Aided Design Integr. Circuits Syst. 22 (2003) 783 (DOI: 10.1109/TCAD.2003.811451).

[11] A. H. El-Maleh: "Test data compression for system-on-a-chip using extended frequency-directed run-length code," IET Comput. Digit. Tech. 2 (2008) 155 (DOI: 10.1049/iet-cdt:20070028).

[12] A. Chandra and K. Chakrabarty: "A unified approach to reduce SOC test data volume, scan power and testing time," IEEE Trans. Comput.-Aided Design Integr. Circuits Syst. 22 (2003) 352 (DOI: 10.1109/TCAD.2002.807895).

[13] M. Nourani and M. H. Tehranipour: "RL-Huffman encoding for test compression and power reduction in scan application," ACM Trans. Des. Autom. Electron. Syst. 10 (2005) 91 (DOI: 10.1145/1044111.1044117).

[14] I. Hamzaoglu and J. H. Patel: "Test set compaction algorithms for combinational circuits," IEEE Trans. Comput.-Aided Design Integr. Circuits Syst. 19 (2000) 957 (DOI: 10.1109/43.856980).

[15] H. K. Lee and D. S. Ha: "HOPE: An efficient parallel fault simulator for synchronous sequential circuits," IEEE Trans. Comput.-Aided Design Integr. Circuits Syst. 15 (1996) 1048 (DOI: 10.1109/43.536711).

[16] R. Sankaralingam, et al.: "Static compaction techniques to control scan vector power dissipation," Proc.18th IEEE VLSI Test Symposium (2000) 35 (DOI: 10.1109/VTEST.2000.843824).

\section{Introduction}

With the rapid development of IC (Integrated Circuit) fabrication processing, an increasing number of transistors are integrated into one single chip, however, it causes multiple increase of test data volume, which not only increases the testing time but also exceeds the tester memory capacity [1]. Compressing test data is an effective approach to reduce test data volume.

The goal of test data compression is to reduce the number of binary bits in original test data which can be generated by the test generation tool after circuit design. The compressed test set is stored in the automatic test equipment (ATE) and a decompress circuit on chip is used to decompress the test data and apply it to the circuit under test (CUT), which is shown in Fig. 1 [2]. The decompress circuit is corresponding to the compression approach and is suitable for all the original test set which using this compression approach.

The test data compression approaches can generally be classified into three categories: code-based schemes, linear-decompression-based schemes and broadcast-scan-based schemes [3]. Code-based schemes mainly aim at given test sets, in which original test data are divided into different symbols and each symbol is replaced by a code word to form the compressed data. We don't need to understand 


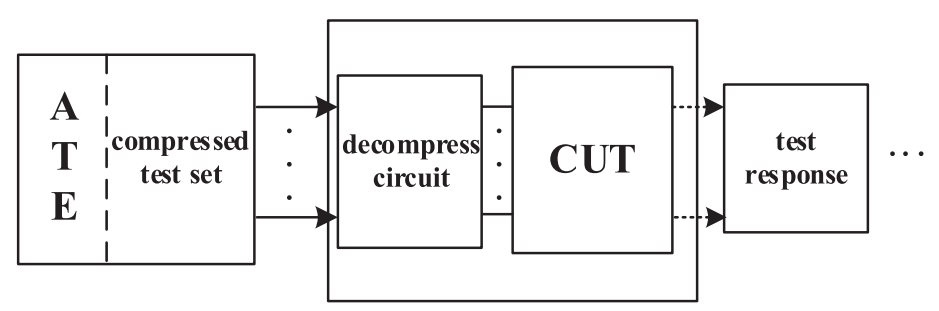

Fig. 1. Test compression structure

the internal structure information of the circuit under test, in addition, the fault simulation and test generation are not needed. Therefore, these schemes are very suitable for test data compression with embedded IP core circuits. According to the differences of symbol division, the coding methods can be classified into two categories. If the scheme encodes a fixed number of input bits, it belongs to the "fixed" category. Similarly, schemes that encode a variable number of input bits are classified under the "variable" category. Huffman coding is the best example of "fixed" scheme. The idea with a Huffman code is to encode symbols that occur more frequently with shorter code words and symbols that occur less frequently with longer code words, such as selective Huffman coding (SHC) [4] and optimal selective Huffman coding (OSHC) [5]. Other "fixed" schemes include dictionarybased coding [6] and block merging coding [7] and so on. Variable categories mainly include two methods: single run-length coding method and double runlength coding method. Single run-length code compression techniques are based on encoding runs of $0 \mathrm{~s}$ such as Golomb code [8], frequency-directed run-length (FDR) code [9] and variable input Huffman code (VIHC) [10]. Double run-length code compression techniques are based on encoding both runs of $0 \mathrm{~s}$ and runs of $1 \mathrm{~s}$ such as extended FDR code (EFDR) [11], alternating run-length coding (AFDR) [12] and mixed double run-length and Huffman coding (RL-HC) [13].

Many bits in test set are don't care bits, which can be filled with any value ( 0 or 1) without decreasing the fault coverage of CUT. In fact, when don't care bits are filled, many faults will be repeatedly tested by multiple vectors [1]. Based on this, a new approach to improve the compression ratio of test set is proposed in this paper, which reverses some specified bits of test set after the don't care bits are filled. This bit reversion-based approach can make test data easier to be compressed without decreasing the fault coverage. The experimental results indicate that our approach can improve the compression ratio of coding by around $10 \%$.

In this paper, Section 2 introduces some typical coding methods. Section 3 presents the theories and algorithms of test set reversion. Section 4 demonstrates the experimental results. Section 5 concludes the whole paper.

\section{Introduction of coding methods}

This section introduces three representative coding methods, i.e., FDR code, EFDR code and Optimal SHC, which come from single run-length coding methods, double run-length coding methods and "fixed" schemes respectively.

\section{1) FDR code}

Run length refers to the sequence composed by the continuous same symbols. FDR code is based on 0 run length, which means the continuous 0 with a tail 1 . In 
FDR code, code words are constituted by the prefix and tail of the same length. In group $\mathrm{A}_{i}$, the length of prefix is $i$, and the prefix of each group represents the run length of the first code of this group. From Group $\mathrm{A}_{i}$ to $\mathrm{A}_{i+1}$, the length of prefix and tail increases one bit respectively. The size of the $i$ th group is equal to $2 i$, that is, group $\mathrm{A}_{i}$ contains $2 i$ members. Run length of $j$ is mapped to group $\mathrm{A}_{i}$, where $i=\left\lceil\log _{2}(j+3)\right\rceil-1$. The FDR code word is shown in Table I.

Table I. FDR code

\begin{tabular}{|c|c|c|c|c|}
\hline Group & Run-length & Group prefix & Tail & Code word \\
\hline \multirow{2}{*}{$\mathrm{A}_{1}$} & 0 & \multirow{2}{*}{0} & 0 & 00 \\
\hline & 1 & & 1 & 01 \\
\hline \multirow{4}{*}{$\mathrm{A}_{2}$} & 2 & \multirow{4}{*}{10} & 00 & 1000 \\
\hline & 3 & & 01 & 1001 \\
\hline & 4 & & 10 & 1010 \\
\hline & 5 & & 11 & 1011 \\
\hline \multirow{4}{*}{$\mathrm{A}_{3}$} & 6 & \multirow{4}{*}{110} & 000 & 110000 \\
\hline & 7 & & 001 & 110001 \\
\hline & $\ldots$ & & $\ldots$ & $\ldots$ \\
\hline & 13 & & 111 & 110111 \\
\hline$\ldots$ & $\ldots$ & $\ldots$ & $\ldots$ & $\ldots$ \\
\hline
\end{tabular}

An example of FDR code is illustrated. For test set $T=\{000000011$ $000000001000001\}$, FDR code is applied to obtain $\mathrm{T}_{\mathrm{FDR}}=\{11000100$ $1100101011\}$, which reduces 6 bits. From this example, it can be observed that the efficiency of FDR code is mainly decided by the quantity of 1 in the test set. The fewer the quantity of 1 is, the fewer the test data bits after coding will be.

\section{2) EFDR code}

In order to encode both runs of $0 \mathrm{~s}$ and $1 \mathrm{~s}$, EFDR code adds one bit based on FDR code to identify the run length is 0 or 1.0 run length means the continuous 0 with a tail 1 , while 1 run length means the continuous 1 with a tail 0 . The EFDR code word is shown in Table II.

For test set $\mathrm{T}=\{01001111111000000001\}$, EFDR code is utilized to obtain the result of $\mathrm{T}_{\mathrm{EFDR}}=\{000001110110110000\}$, which reduces 2 bits. From this example, it can be observed that when the binary bit of the test set flips (from 0 to 1 or from 1 to 0 ), there will be a run length. When the flip number is fewer in a test set, the compression ratio will be higher.

\section{3) Optimal SHC}

Optimal SHC belongs to the coding method from fixed length to variable length, which encodes the data blocks of certain length with variable-length code words. Data blocks with high frequency are distributed with short code words and vice versa so as to achieve the purpose of data compression. In order to reduce the hardware cost, data blocks with high frequency apply Huffman coding while the ones with low frequency will not be encoded but introduced by ATE directly. 
Table II. EFDR code

\begin{tabular}{|c|c|c|c|c|c|}
\hline Group & $\begin{array}{l}\text { Run- } \\
\text { length }\end{array}$ & $\begin{array}{l}\text { Group } \\
\text { prefix }\end{array}$ & Tail & $\begin{array}{l}\text { Code word } \\
\text { runs of } 0 \mathrm{~s}\end{array}$ & $\begin{array}{l}\text { Code word } \\
\text { runs of } 1 \mathrm{~s}\end{array}$ \\
\hline \multirow{2}{*}{$\mathrm{A}_{1}$} & 1 & \multirow{2}{*}{0} & 0 & 000 & 100 \\
\hline & 2 & & 1 & 001 & 101 \\
\hline \multirow{4}{*}{$\mathrm{A}_{2}$} & 3 & \multirow{4}{*}{10} & 00 & 01000 & 11000 \\
\hline & 4 & & 01 & 01001 & 11001 \\
\hline & 5 & & 10 & 01010 & 11010 \\
\hline & 6 & & 11 & 01011 & 11011 \\
\hline \multirow{4}{*}{$\mathrm{A}_{3}$} & 7 & \multirow{4}{*}{110} & 000 & 0110000 & 1110000 \\
\hline & 8 & & 001 & 0110001 & 1110001 \\
\hline & $\ldots$ & & $\ldots$ & $\ldots$ & $\ldots$ \\
\hline & 14 & & 111 & 0110111 & 1110111 \\
\hline$\ldots$ & $\ldots$ & $\ldots$ & $\ldots$ & $\ldots$ & $\ldots$ \\
\hline
\end{tabular}

The following example will illustrate the process of optimal SHC. In Table III, the test set $\mathrm{T}$ is composed by 5 vectors of 16 in length. After dividing the test set $\mathrm{T}$ into data blocks of 4 in length, the second column and third column in the table list each category of data blocks and their frequencies. For example, if only the data blocks with the first three highest frequencies are encoded, the frequency of uncoded blocks will be $2 / 20+1 / 20=3 / 20$. Subsequently, the data blocks with the highest frequencies like 1010, 0000, 1111 and uncoded blocks will apply Huffman coding, which is shown in Fig. 2. The data block 1010 with the highest frequency will be represented with only one bit of 0 , while the uncoded blocks need to add the identifying bits of 111 before the data block (such as encoding 0001 into 1110001 ), which will be 7 bits. The compression ratio in this case is equal to $38.8 \%$. Therefore, it can be concluded that the fewer the uncoded data blocks in test set are, the higher the compression ratio will be.

Table III. Division of test set and frequency of different blocks

\begin{tabular}{|c|c|c|}
\hline Test set $\mathrm{T}$ & Different blocks & Frequency \\
\hline 1010000010101111 & 1010 & $9 / 20$ \\
\cline { 2 - 3 } 1111000010100001 & 0000 & $5 / 20$ \\
\cline { 2 - 3 } 1010000000101010 & 1111 & $3 / 20$ \\
\cline { 2 - 3 } 0000101010100000 & 0001 & $2 / 20$ \\
\cline { 2 - 3 } 1010111110100001 & 0010 & $1 / 20$ \\
\hline
\end{tabular}

\section{Bit reversion theories and algorithms}

To understand the bit reversion theories of test set, the concept of test redundancy must be defined first.

Definition 1: In test set $\mathrm{T}$, the quantity of test vectors which can detect a specified fault is called the test redundancy of this fault. The average value of test redundancy of all faults is called as the average test redundancy rate of test set $\mathrm{T}$. 


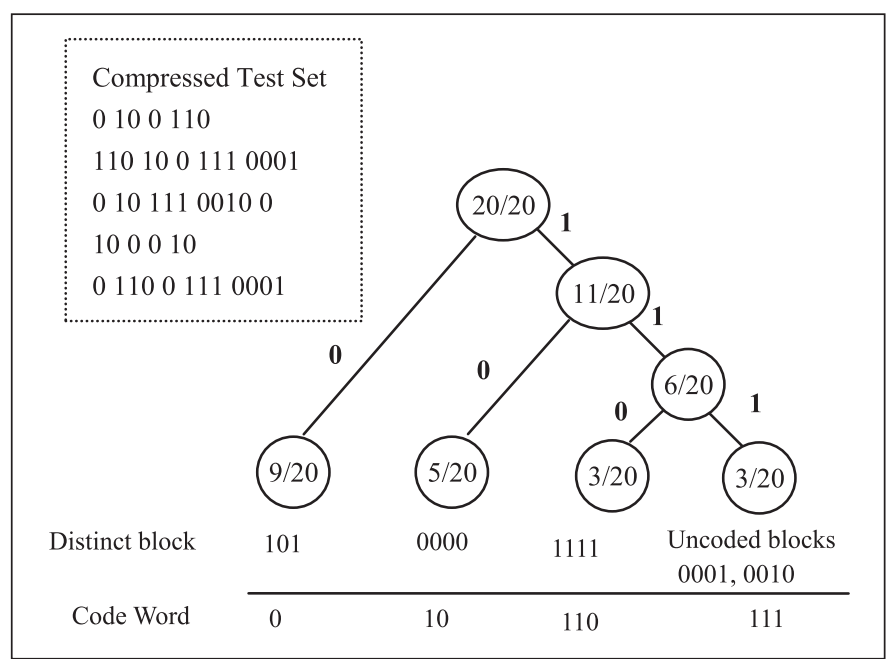

Fig. 2. Optimal SHC

The average test redundancy reflects the degree of a single fault in a circuit being repeatedly detected by test set $\mathrm{T}$. Table IV illustrates the test redundancy rate before and after X (don't care bits) filling.

Table IV. Average test redundancy rate before and after $\mathrm{X}$ filling

\begin{tabular}{|c|c|c|}
\hline Circuit & X not filled & X filled with random bits \\
\hline s5378 & 8.6 & 24.9 \\
\hline s9234 & 8.8 & 26.4 \\
\hline s13207 & 7.5 & 53.8 \\
\hline s15850 & 7 & 27.6 \\
\hline s38417 & 7.5 & 22.1 \\
\hline s38584 & 6.2 & 32.1 \\
\hline Average & 7.6 & 31.2 \\
\hline
\end{tabular}

It can be observed that after $\mathrm{X}$ filling in the test set, the test redundancy will increase. Some specified bits in the test set can be reversed without decreasing the fault coverage because of the test redundancy. When the test set changes to benefit the compression, the compression ratio will be improved.

FDR code is taken as the example to illustrate the basic process of test set reversion. As section 2 has mentioned, the purpose of test set reversion is to reduce the number of 1 . The algorithm 1 illustrates the basic process of test set reversion: For each $1 \mathrm{~s}$ in the test set $\mathrm{T}$, change it to $0 \mathrm{~s}$ if the fault coverage does not reduce.

Algorithm 1. TestSetReversion

Coverage $\leftarrow$ FaultCoverage $(\mathrm{T}) / /$ solve fault coverage of $\mathrm{T}$

FOR bit IN T//bit is a certain bit in $\mathrm{T}$

IF bit $=1 \quad / /$ turn 1 of $\mathrm{T}$ into 0

bit $\leftarrow 0$

//if the fault coverage is affected, restore

IF Coverage $\neq$ FaultCoverage(T)

$$
\text { bit } \leftarrow 1
$$


Factors affecting the test set reversion ability include average redundancy, distribution of test redundancy and bit reversion method. Different reversion strategies are needed for different coding methods. Three general algorithms will be listed as follows.

\section{1) Single run-length reversion algorithm}

The purpose of bit reversion for single run-length coding method is to reduce the quantity of $1 \mathrm{~s}$ in the test set.

Definition 2: Reverse all the bits 1 of a column in test set successively according to a certain order. If the fault coverage of the whole test set after reversing the bit is not affected, this bit will be reversed. The quantity of reversed bits is called the reversion weight of the column (RWC).

Algorithm 2. SingleRunLengthReversion

WHILE T not empty //when test set $\mathrm{T}$ is not empty

$/{ }^{*} \mathrm{~m}$ column is randomly selected from $\mathrm{T}$ to find out the maximum column of reversion right*/

$$
\begin{aligned}
& \text { MaxColumn } \leftarrow 0 \\
& \text { MaxWeight } \leftarrow 0 \\
& \text { FOR } i \leftarrow 1 \text { to } m \\
& \text { Column }=\text { RandomChoose }(\mathrm{T}) \\
& \text { IF MaxWeight }<\text { weight }(\text { Column }, 0) \\
& \text { MaxColumn } \leftarrow \text { Column } \\
& \text { MaxWeight } \leftarrow \text { weight }(\text { Column }, 0)
\end{aligned}
$$

$/{ }^{*} \mathrm{n}$ types of reversion order is formed randomly to find out the maximum order of reversion right */

$$
\begin{aligned}
& \text { MaxOrder } \leftarrow 0 \\
& \text { MaxWeight } \leftarrow 0 \\
& \text { FOR } j \leftarrow 1 \text { to } n \\
& \text { Order }=\text { RandomOrder }() \\
& \text { IF MaxWeight }<\text { weight }(\text { MaxColumn, Order }) \\
& \quad \text { MaxOrder } \leftarrow \text { Order } \\
& \quad \text { MaxWeight } \leftarrow \text { weight (MaxColumn, Order })
\end{aligned}
$$

$/{ }^{*}$ find the proper column and reversion order and take this column as this order for reversion and mark it as reversed. $/$

\section{ReverseColumn(MaxColumn, MaxOrder) \\ Remove(MaxColumn)}

The single run-length reversion algorithm is shown as algorithm 2. Firstly, pick up randomly $m$ columns from the test set, and selects among of them the column with the maximal RWC. This column is called the MaxColumn. Secondly, for the MaxColumn generate randomly $n$ different orders and select an order in the way that the maximal RWC can be obtained if the flips are conducted. This order is called the MaxOrder. Finally, reverse all the bits 1 for the MaxColumn in the order of the MaxOrder, and sign subsequently the MaxColumn that has been reversed. The three steps are repeated until all the bits 1 in the test set are tried.

\section{2) Double run-length reversion algorithm}

The purpose of test set reversion for double run-length coding method is to reduce the quantity of flips. A consecutive sequence of equal bits is called non-flip 


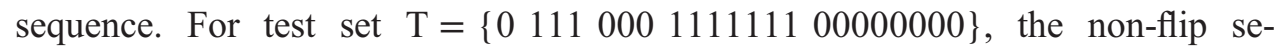
quences are $0,111,000,1111111$ and 00000000 . The shortest non-flip sequence will be firstly reversed to reduce the flip number, and then $T=\{\underline{1} 111000$ $111111100000000\}$. If the fault coverage of test set $\mathrm{T}$ does not change, we continue to reverse the shortest non-flip sequence at present, get $T=\left\{\begin{array}{lll}1 & 111\end{array}\right.$ $111111111100000000\}$. The whole algorithm is shown in algorithm 3.

Algorithm 3. DoubleRunLengthReversion

Coverage $\leftarrow$ FaultCoverage $(\mathrm{T}) / /$ solve the fault coverage

FOR $i \leftarrow 1$ to $m \quad / /$ reverse the flip string with length less than $\mathrm{i}$

FOR NotFlipString IN T // flip string in $\mathrm{T}$

If length(NotFlipString) $<i$

Reverse(NotFlipString) // reverse flip string

//if the fault coverage is affected, restore

IF Coverage $\neq$ FaultCoverage $(\mathrm{T})$

Reverse(NotFlipString)

\section{3) "Fixed" reversion algorithm}

"Fixed" scheme divides test set into blocks of the same fixed length for coding respectively. There are many types of "fixed" scheme, but there is one common point: In coding process, the current data block is compared with the target blocks. If they are equal, a corresponding code word will be assigned to the current block, if not, extra bits will be added in front of the current block to distinguish it from the code words. Therefore, for "fixed" scheme, the purpose of reversion is to make the number of uncoded blocks smaller while applying short code words for coded blocks.

The reversion algorithm is shown in algorithm 4. For "fixed" schemes, the test set $\mathrm{T}$ is divided into blocks with fixed length. All of the blocks are grouped into one set, named B. Similarly, the "target blocks" are grouped into a set, named D. For each blocks in B, it will be replaced with one of the target blocks in D, if the replace do not change the fault coverage. The target blocks are updated after every loop. This algorithm is repeated until no blocks can be replaced.

Algorithm 4. BlockReversion

Coverage $\leftarrow$ FaultCoverage $(\mathrm{T}) / /$ get the fault coverage of $\mathrm{T}$

$\mathrm{D}=$ GetDestBlock ()$\quad / /$ get the original target block $\mathrm{D}$

$/ /$ for each block in B

$/ /$ replace each block of dest in D without affecting the fault coverage ratio FOR block IN $B$

FOR dest IN D //target block

block $\leftarrow$ dest

IF Coverage $=$ FaultCoverage $(\mathrm{T}) / /$ find suitable block BREAK

//if no suitable block is found in D, restore the block

IF Coverage $\neq$ FaultCoverage $(\mathrm{T})$

recovery (block)

update(D) // update target block set 


\section{Experimental results}

We implemented the bit reversion algorithm using $\mathrm{C}++$ and verified its effectiveness on the ISCAS' 89 benchmark circuits. Six largest circuits are selected as the experimental circuits, and the test sets are generated by the Mintest ATPG [14]. Fault simulator "HOPE" [15] is used for fault simulation. Certainly, the test set will be generated by some specific methods during test phase after the design for every actual circuit, and our approach can be suitable for any given original test set. Moreover, bit reversion-based approach has an advantage that requires no new decompress circuit, but uses the existing decompress circuit corresponding to such as FDR, EFDR coding methods and so on. As a result, there is no additional overhead in the process of decompression.

Table $\mathrm{V}$ compares the compression ratio changes of three single run-length coding methods before and after reversion (Golomb [8], FDR [9], VIHC [10]). The first column is circuit name, the $2-4$ columns are the compression ratio of original test set, the 5-7 columns are the compression ratio of test set after reversion. It can be observed from the table that the compression ratio has improved $7.42 \% \sim 11.20 \%$ after reversion.

Table V. Compression ratio comparison of single run-length coding methods

\begin{tabular}{|c|c|c|c|c|c|c|}
\hline \multirow{2}{*}{ Circuit } & \multicolumn{3}{|c|}{ Original } & \multicolumn{3}{c|}{ Test set reversion } \\
\cline { 2 - 7 } & Golomb & FDR & VIHC & Golomb & FDR & VIHC \\
\hline s5378 & 37.11 & 47.98 & 51.78 & 48.63 & 57.83 & 60.52 \\
\hline s9234 & 45.25 & 43.61 & 47.25 & 53.63 & 50.36 & 55.06 \\
\hline s13207 & 79.74 & 81.3 & 83.51 & 83.03 & 83.40 & 85.86 \\
\hline s15850 & 62.82 & 66.21 & 67.94 & 70.55 & 72.37 & 74.49 \\
\hline s38417 & 28.37 & 43.37 & 53.36 & 57.27 & 62.69 & 67.19 \\
\hline s38584 & 57.17 & 60.93 & 62.28 & 64.56 & 65.60 & 67.55 \\
\hline Average & 51.74 & 57.23 & 61.02 & $\mathbf{6 2 . 9 4}$ & $\mathbf{6 5 . 3 8}$ & $\mathbf{6 8 . 4 4}$ \\
\hline
\end{tabular}

As shown in Table VI, after bit reversion, the compression ratio of double runlength reversion has improved 8.42\% 12.07\% (AFDR [12], EFDR [11], RL-HC [13]). The double run-length reversion algorithm can better improve the compression ratio of test set than single run-length reversion algorithm. This is because the single run-length reversion algorithm merely fills don't care bits of test set into 0 , which makes the redundancy distribution uneven and influences the effects of test set reversion.

Table VII shows the results of Optimal SHC [5] for "fixed" scheme. Compared to the previous two methods, the compression ratio of fixed scheme has improved more by $12.7 \%$ with the final compression ratio of $81.22 \%$, exceeding the majority of code-based compression methods.

Finally, we compare test power [16] between original test set and test set after 
Table VI. Compression ratio comparison of double run-length coding methods

\begin{tabular}{|c|c|c|c|c|c|c|}
\hline \multirow{2}{*}{ Circuit } & \multicolumn{3}{|c|}{ Original } & \multicolumn{3}{c|}{ Test set reversion } \\
\cline { 2 - 7 } & AFDR & EFDR & RL-HC & AFDR & EFDR & RL-HC \\
\hline s5378 & 50.77 & 53.67 & 53.75 & 63.36 & 63.51 & 65.89 \\
\hline s9234 & 44.96 & 48.66 & 47.59 & 62.04 & 61.34 & 66.07 \\
\hline s13207 & 80.23 & 82.49 & 82.51 & 85.91 & 85.74 & 88.63 \\
\hline s15850 & 65.83 & 68.66 & 67.34 & 76.02 & 75.85 & 78.65 \\
\hline s38417 & 60.55 & 62.02 & 64.17 & 69.95 & 69.49 & 72.38 \\
\hline s38584 & 61.13 & 64.28 & 62.40 & 75.24 & 74.39 & 78.55 \\
\hline Average & 60.58 & 63.30 & 62.96 & $\mathbf{7 2 . 0 9}$ & $\mathbf{7 1 . 7 2}$ & $\mathbf{7 5 . 0 3}$ \\
\hline
\end{tabular}

average power consumption and peak power reduce by around $9 \%$, while double run-length reversion algorithm makes those reduce by almost a half. This is because that double run-length reversion algorithm aims to reduce the number of bit flips while the power consumption is produced due to the bit flips of test set. The average power consumption of Optimal SHC increases by $25.73 \%$ but the peak power remains almost the same. In fact, the power consumption of "fixed" schemes can be controlled, which needs to select the blocks with few flips as the target blocks, and this will reduce some compression ratio of "fixed" schemes.

Table VII. Compression ratio comparison of "fixed" scheme

\begin{tabular}{|c|c|c|}
\hline \multirow{2}{*}{ Circuit } & Original & Test set reversion \\
\cline { 2 - 3 } & Optimal SHC & Optimal SHC \\
\hline $\mathrm{s} 5378$ & 59.60 & 74.94 \\
\hline $\mathrm{s} 9234$ & 60.00 & 77.58 \\
\hline $\mathrm{s} 13207$ & 85.30 & 89.75 \\
\hline $\mathrm{s} 15850$ & 72.20 & 82.77 \\
\hline $\mathrm{s} 38417$ & 64.50 & 76.77 \\
\hline $\mathrm{s} 38584$ & 69.50 & 85.52 \\
\hline Average & 68.52 & $\mathbf{8 1 . 2 2}$ \\
\hline
\end{tabular}

Table VIII. Comparison of power consumption

\begin{tabular}{|c|c|c|c|c|c|c|}
\hline \multirow{2}{*}{ Test set } & \multicolumn{2}{|c|}{ Single run-length } & \multicolumn{2}{c|}{ Double run-length } & \multicolumn{2}{c|}{ Optimal SHC } \\
\cline { 2 - 7 } & Average & Peak & Average & Peak & Average & Peak \\
\hline Original & 58281 & 207116 & 38242 & 177852 & $\mathbf{1 1 1 0 8 8}$ & 193153 \\
\hline Reversed & $\mathbf{5 3 0 8 8}$ & $\mathbf{1 8 7 2 6 8}$ & $\mathbf{2 2 8 1 9}$ & $\mathbf{7 3 6 6 0}$ & 139670 & $\mathbf{1 8 6 6 0 1}$ \\
\hline
\end{tabular}

\section{Conclusion}

We propose a new test data compression approach based on bit reversion, which makes it easier to compress data by reversing some bits of the test set without decreasing their fault coverage. Although the use of bit reversion-based approach 
requires the fault simulation process, a higher compression rate can be achieved. Therefore, more storage space and more test application time can be saved in the process of testing a large number of chips. From this point of view, our approach has very important practical significance.

In order to fully verify the effectiveness of our approach, we propose some general algorithms for three types of coding methods. The experimental results indicate that the proposed approach can greatly improve the compression ratio of test data, and reduce test power consumption to a certain extent.

Besides code-based schemes, linear-decompression-based schemes and broadcast-scan-based schemes can also apply our approach.

\section{Acknowledgments}

This paper was supported in part by the National Natural Science Foundation of China (NSFC) under grant No. 61504013 and in part by Hunan Province Undergraduates Innovating Experimentation Project of China under grant No. (2016)283-192. 